\title{
DIABETES
}

\section{Protective role of autophagy in pancreatic $\beta$ cells}

$\mathrm{T}$ he results of three studies published simultaneously in The Journal of Clinical Investigation highlight the importance of autophagy in protecting the function of pancreatic $\beta$ cells and preventing the development of type 2 diabetes mellitus (T2DM).

"For many years it was proposed that T2DM was caused by insulin resistance, and insulin resistance was attributed to obesity," explains Peter Butler, senior investigator in one of the studies. "The problem with this model is that $80 \%$ of individuals who have morbid obesity never develop T2DM, they simply adapt to the insulin resistance by appropriately increasing insulin secretion to maintain normal blood sugar levels." How T2DM develops in people with insulin resistance, therefore, still warrants clarification.

Earlier findings in mice showed that autophagy is important to maintain $\beta$-cell mass and function and revealed a link between impaired autophagy and susceptibility to development of T2DM. However, differences in the pathogenesis of T2DM are likely to exist between mice and men. "One of the hallmarks of human T2DM is the deposition of amyloid," say Yoshio Fujitani and Hirotaka Watada, senior researchers of the second study. Islet amyloid is derived from islet amyloid polypeptide (IAPP, also known as amylin), which is co-secreted with insulin by pancreatic islets. Islet amyloid deposits characterize T2DM in humans, whereas IAPP in mice does not form amyloid aggregates. Intracellular oligomeric forms of IAPP are toxic to $\beta$ cells, whereas extracellular amyloid is thought to be fairly inert. "Amyloidogenic proteins such as human IAPP are cleared preferentially by autophagy, whereas nonamyloidogenic proteins such as murine IAPP are efficiently cleared by proteasomal degradation," explains Myung-Shik Lee, senior investigator in the third study.

All of the studies involved investigation of the relationship between autophagy deficiency and the pathogenesis of human

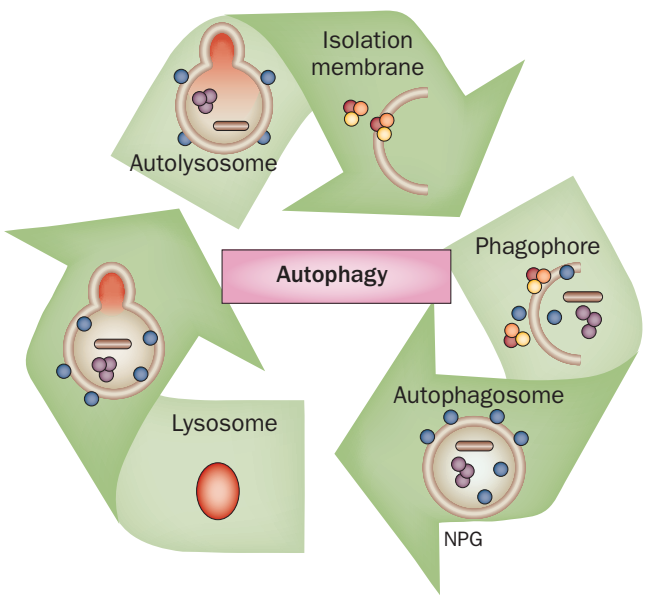

T2DM by analysis of transgenic mice expressing human IAPP. The Butler and Lee teams showed that specific expression of human IAPP in $\beta$ cells of transgenic mice does not lead to the development of T2DM per se. However, mice that overexpress human IAPP develop T2DM when autophagy is impaired in $\beta$ cells. Fujitani, Warada and colleagues studied a different mouse model with a human IAPP knockin. These mice also did not develop T2DM, but glucose tolerance deteriorated when a $\beta$-cell-specific defect in autophagy was introduced. In the three studies, concomitant human IAPP expression and impaired autophagy in $\beta$ cells were associated with $\beta$-cell apoptosis and loss of $\beta$-cell mass.

The results of the studies led by Butler and Fujitani and Watada further indicate that the p62 scaffold protein has a role in sequestering misfolded human IAPP and stopping release of toxic oligomers, at least temporarily. "This process might be how amyloid fibrils are formed in other amyloid-related diseases," Butler speculates. Butler and colleagues also observed that $\beta$-cell apoptosis in autophagy-deficient mice expressing human IAPP seems to be due to loss of protection against the effects of reactive oxygen species.

Lee and his group used trehalose, a substance reported to enhance autophagy, in autophagy-deficient mice with human IAPP $\beta$-cell expression that were fed a high-fat diet. Various metabolic features improved with treatment, including glucose levels and glucose tolerance. "These results are reminiscent of previous data indicating that autophagy enhancers can ameliorate neurologic symptoms in mice with neurodegenerative disorders showing accumulation of amyloid-prone proteins," comments Lee.

Following their observation of a correlation between levels of autophagic activity in $\beta$ cells and $\beta$-cell function, Fujitani, Watada and colleagues would like to develop a biomarker by which autophagic status can be assessed noninvasively. "We also need to address the molecular mechanisms whereby autophagy is deficient in $\beta$ cells in humans and animals with T2DM," they add.

"We already know that in people who are vulnerable to T2DM (such as those with a family history of diabetes), a lifestyle that places less burden on their $\beta$ cells (regular physical activity, moderate consumption of dietary fats) reduces the risk of developing T2DM," comments Butler. "The new data raise hopes that therapeutic approaches that enhance misfolded protein clearance might be developed both for T2DM and for neurodegenerative diseases." Lee and co-workers are planning to develop autophagy enhancers for the treatment of human T2DM characterized by amyloid deposition in pancreatic islets, in addition to investigating the mechanisms of human IAPP oligomer and amyloid formation.

Joana Osório 